\title{
Early Medicaid Expansions and Drug Overdose Mortality in the USA: a Quasi-experimental Analysis
}

\author{
Atheendar S. Venkataramani, MD, $P h D^{1,2}$ and Paula Chatterjee, MD, MPH $H^{2,3}$ \\ 'Department of Medical Ethics and Health Policy, Perelman School of Medicine, University of Pennsylvania, Philadelphia, PA, USA; ${ }^{2}$ Leonard Davis \\ Institute of Health Economics, University of Pennsylvania, Philadelphia, PA, USA; ${ }^{3}$ Division of General Internal Medicine, Perelman School of \\ Medicine, University of Pennsylvania, Philadelphia, PA, USA.
}

KEY WORDS: drug overdose mortality; opioid crisis; Medicaid; USA; quasiexperimental.

$\mathrm{J}$ Gen Intern Med 34(1):23-5

DOI: $10.1007 / \mathrm{s} 11606-018-4664-7$

(c) Society of General Internal Medicine 2018

\section{INTRODUCTION}

The widespread availability of prescription and illicit opioids is thought to be a key driver of the rise in drug overdose mortality in the USA. ${ }^{1}$ The role of health insurance in increasing access to prescription opioids has thus emerged as a key policy issue. Policymakers and commentators have raised concerns that Medicaid expansions - such as those implemented as part of the Affordable Care Act (ACA) - may have caused the rise in drug overdose mortality, citing more deaths from drug overdose in states that expanded Medicaid versus those that did not. ${ }^{2}$

Assessing whether insurance expansions helped cause the rise in drug overdose mortality is challenging in the midst of a national crisis. The availability of opioids ${ }^{1}$ and drug overdose mortality rates ${ }^{3}$ were already increasing prior to the ACA. In addition, the crisis has evolved like an epidemic and assessing root causes is difficult during periods of accelerating growth in disease rates.

We examined the effect of Medicaid expansions on drug overdose mortality using early Medicaid expansions in Arizona, Maine, and New York from 2001 to 2002. All three states expanded coverage up to $100 \%$ of the federal poverty line for childless adults. Arizona and New York further expanded coverage to $200 \%$ and $150 \%$ of the federal poverty line, respectively, for parents with dependent children. These expansions occurred on the eve of the national rise in drug overdose mortality, making it possible to investigate whether insurance expansions served as an underlying cause.

\section{METHODS}

We used publicly available, state-level data on ageadjusted, drug overdose mortality rates (ICD 10: X40X44, X60-X64, X85, Y10-Y14) from 1999 to 2008 for 25-64 year-olds from the US Centers for Disease

Published online September 20, 2018
Control (https://wonder.cdc.gov/mortSQL.html). Like prior work, ${ }^{3}$ we focused on all drug overdose deaths given the well-known underreporting of opioid-attributable deaths and the fact that over $60 \%$ drug overdose deaths are likely attributable to opioids. ${ }^{4}$ The starting year (1999) corresponded to the beginning of the ICD-10 era, while the ending year (2008) was chosen on the basis of prior work examining the health consequences of the same early Medicaid expansions. ${ }^{5}$

We estimated difference-in-differences models comparing changes in drug overdose mortality in early expansion states versus non-expansion states. We adjusted for state-fixed effects to account for time-invariant differences between states; year-fixed effects to adjust for national secular trends; and poverty and unemployment rates (obtained from the University of Kentucky Center for Poverty Research, UKCPR; http://www.ukcpr.org/data). Models were weighted by population and standard errors were corrected for state-level. Replication data, code, and other materials are available in the Harvard Dataverse (https://dataverse.harvard.edu/dataset.xhtml? persistentId=doi:10.7910/DVN/9ZS4KR).

\section{RESULTS}

Unadjusted gaps in drug overdose mortality rates were stable between expansion and non-expansion states prior to Medicaid expansions, but grew faster in nonexpansion states thereafter (Fig. 1a). A similar pattern was seen when restricting control states to nonexpanding neighbors (New Hampshire, Pennsylvania, New Mexico, Nevada, Fig. 1b).

Difference-in-differences estimates (Table 1) confirmed this pattern. Drug overdose mortality rates were, on average, lower by 3.7 deaths per 100,000 per year in expansion states relative to non-expansion states after policy implementation through $2008(\beta=-3.7$; $95 \%$ CI, $-5.6,-1.9 ; p<0.001)$. Estimates were similar after additional adjustment for state-specific linear time-trends (to account for differential pre-policy trends) and other social policy measures (state minimum wage, welfare and food stamp programs generosity, and state 

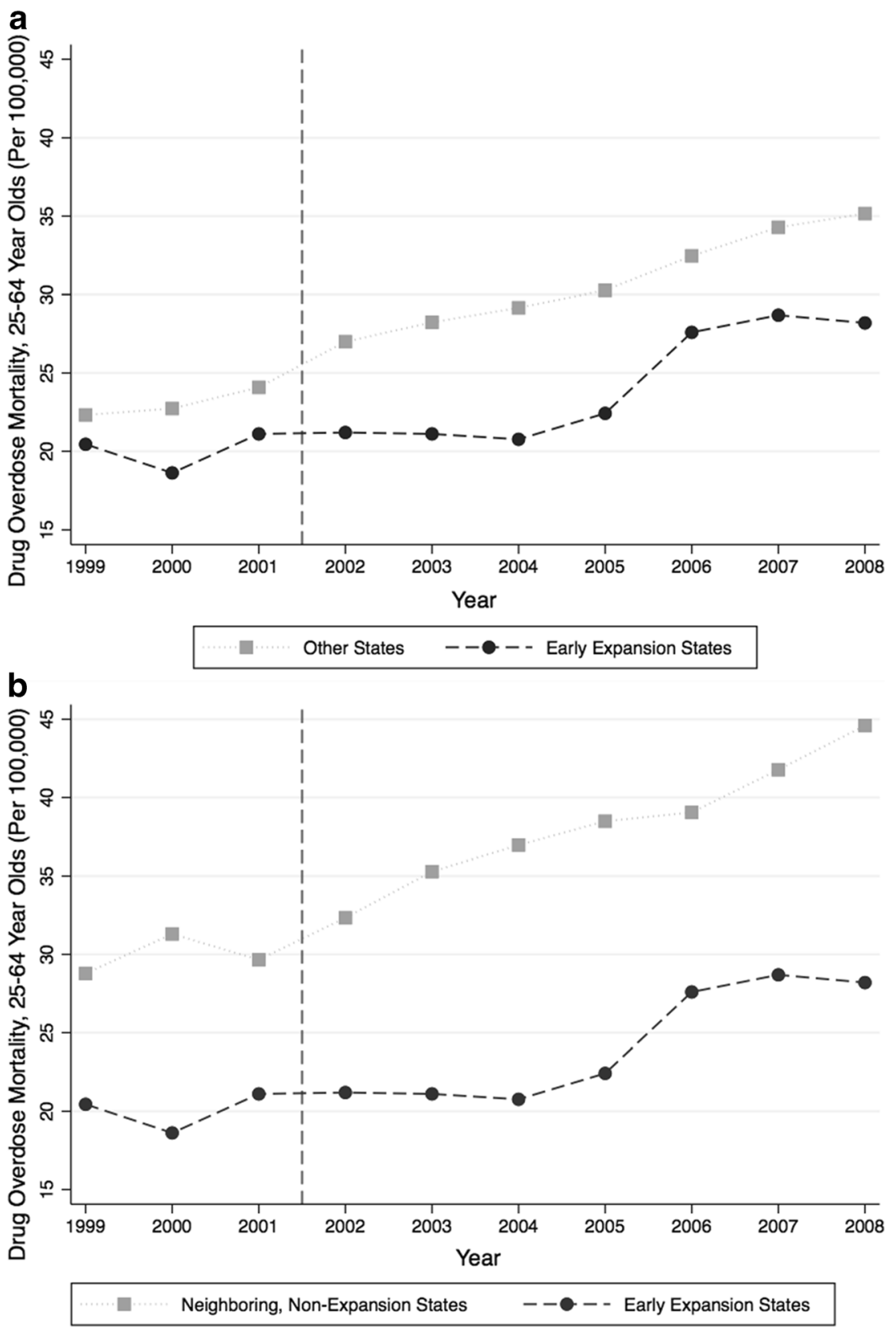

Figure 1 Trends in drug overdose mortality. Notes: drug overdose mortality rates for 25-64 year-olds (age adjusted, per 100,000) for states expanding Medicaid early (Arizona, Maine, New York) versus (a) all other states (plus District of Columbia) and (b) non-expanding, neighboring states (New Hampshire, Pennsylvania, New Mexico, Nevada). The vertical line at 2002 represents the first year after Medicaid expansion (Arizona and New York expanded in late 2001, Maine in late 2002).

Supplemental Security Income-all obtained from the UKCPR) ( $\beta=-4.0 ; 95 \% \mathrm{CI},-7.8,-0.2 ; p=0.04)$. Restricting the control group to the non-expanding neighboring states also yielded similar results $(\beta=-$ $4.4 ; 95 \% \mathrm{CI},-6.9,-1.3 ; p=0.005)$.

\section{DISCUSSION}

Drug overdose mortality rates rose less sharply in three states that expanded Medicaid in the early 2000s as compared to states that did not. These findings suggest that Medicaid expansions were unlikely to have contributed to the subsequent rise in drug overdose deaths and may, in fact, have been protective.

Limitations of our approach include the small number of expansion states and the difficulty of generalizing findings to subsequent Medicaid expansions, which occurred in the accelerating phase of the epidemic.

Nonetheless, these findings should offer further reassurance to policymakers concerned that Medicaid expansions have caused the rise in drug overdose mortality. The striking results suggest there may be important lessons to be learned from this historical episode. In particular, future work should examine mechanisms 
Table 1 Difference-in-Differences Estimates

\begin{tabular}{llll}
\hline \hline & $\mathbf{( 1 )}$ & $\mathbf{( 2 )}$ & $\mathbf{( 3 )}$ \\
\hline Estimate $(\beta)$ & -3.73 & -3.99 & -4.42 \\
& {$[-5.60,-1.86]$} & {$[-7.82,-0.16]$} & {$[-6.91,-1.93]$} \\
& $p<0.001$ & $p=0.042$ & $p=0.005$ \\
$\mathrm{~N}$ (state-years) & 510 & 510 & 70 \\
$\mathrm{~N}$ (states and & 51 & 51 & 7 \\
D.C.) & & & \\
\hline
\end{tabular}

Notes: Estimates from difference-in-differences least squares models, reflecting the differential change in age-adjusted drug overdose mortality for 25-64 year-olds (per 100,000) in expansion versus nonexpansion states (denoted by $\beta$ ). All models adjust for state fixed effects, year fixed effects, and state-year unemployment and poverty rates and are weighted by state population of 25-64 year-olds. 95\% CI, adjusted for clustering at the state level, in square brackets. Column [2] additionally includes state-specific linear time trends and state-year measures of social program generosity (state minimum wage in dollars per hour, welfare generosity as measured by the monthly maximum benefits from welfare and food stamp programs for a four-person family, and maximum monthly state Supplemental Security Income-all obtained from the UKCPR). Column [3] is identical to Column [1] except here we restricted the control states to non-expanding neighboring states

underlying these findings, which may include improved financial security and mental health upon obtaining insurance, ${ }^{6}$ or increased access to treatment for substance use disorders.
Corresponding Author: Atheendar S. Venkataramani, $M D, P h D$; Department of Medical Ethics and Health Policy, Perelman School of Medicine University of Pennsylvania, Philadelphia, PA, USA (e-mail: atheenv@pennmedicine.upenn.edu).

Funding Information No specific funding was received for this study. ASV reports salary support from the National Institutes of Health (Mentored Career Development Award, grant K23MH106362).

\section{Compliance with Ethical Standards:}

Conflict of Interest: The authors declare that they have no conflict of interest.

\section{REFERENCES}

1. Rudd RA, Seth P, David F, Scholl L. Increases in drug and opioid-involved overdose deaths - United States, 2010-2015. Morb Mortal Wkly Rep. 2016;65(50-51):1445-52.

2. Finley A. Does Medicaid spur opioid abuse? Wall Street J. 2017;Sept. 24. https://www.wsj.com/articles/does-medicaid-spur-opioid-abuse1506289279 .

3. Goodman-Bacon A, Sandoe E. Did Medicaid expansion cause the opioid epidemic? There's little evidence that it did. Health Affairs Blog. 2017.

4. Ruhm CJ. Corrected US opioid-involved drug poisoning deaths and mortality rates, 1999-2015. Addiction. 2018;113:1339-1344.

5. Sommers BD, Baicker K, Epstein AM. Mortality and access to care among adults after state Medicaid expansions. N Engl J Med. 2012;367:1025-34.

6. Sommers BD, Gawande AA, Baicker $\mathbf{K}$. Health insurance coverage and health - what the recent evidence tells us. N Engl J Med. 2017;377:586-93. 